\title{
A PVDF-Based Deformation and Motion Sensor: Modeling and Experiments
}

\author{
Jingang Yi, Senior Member, IEEE, and Hong Liang
}

\begin{abstract}
In this paper, we present the mathematical modeling, analysis, and experiments of a new deformation and motion measurement sensor that is made of polyvinylidene fluoride (PVDF) thin-film. The PVDF-based deformation sensor is designed and fabricated for several applications, such as deformation detection of automotive tires and insect locomotion measurements. In the sensing system, only two ends of the strip-shape sensor are attached to the moving object and under the relative motion of two ends, the sensor is buckled. The design provides a new nonintrusive method of measuring deformation and motion, which are desirable in certain applications. The analytical model of the sensing system is based on the synthesis of an elastica modeling of the PVDF thin-film under buckling motion and a Duhem hysteresis model. The modeling and analysis results are compared and validated with experiments that are conducted on a testing kit.
\end{abstract}

Index Terms-Buckling, hysteresis, insect locomotion, polyvinylidene fluoride (PVDF), tire deformation.

\section{INTRODUCTION}

$\mathbf{P}$ OLYVINYLIDENE FLUORIDE (PVDF)-based sensors have been used for many applications, such as structure health monitoring [1], vibration sensing [2], and in-motion vehicle weight measurement [3], [4]. In this paper, we present the new knowledge and methods of PVDF-based sensor for measuring the deformation and motion in mechanical and biological systems. Two different types of applications motivate us to study the PVDF-based deformation and motion sensing mechanism: one is for real-time automotive tire deformation measurements and the other is for insect locomotion detection.

Tire/road interaction plays an important role for vehicle safe operations [5]. Tread deformation is the critical information for understanding tire/road interactions [6], [7]. For example, in [8], "Darmstadt Tire Sensor" has been presented to measure the tire tread deformation. Surface acoustic wave (SAW) sensors have been proposed for a "smart tire" application in [9]. Capacitance sensors have been developed for monitoring the tire

Manuscript received October 16, 2007; revised December 2, 2007; accepted December 5, 2007. This work was supported in part by Texas Transportation Institute (TTI) and in part by the National Science Foundation (NSF) under Grant IIS-0515930. This paper was presented in part at the 2007 World Forum on Smart Materials and Smart Structure Technology, Nanjing, China, May 25-27, 2007. The associate editor coordinating the review of this paper and approving it for publication was Prof. Fabien Josse.

J. Yi is with the Department of Mechanical Engineering, San Diego State University, San Diego, CA 92182 USA (e-mail: jgyi @mail.sdsu.edu).

$\mathrm{H}$. Liang is with the Department of Mechanical Engineering, Texas A\&M University, College Station, TX 77843 USA (e-mail: hliang@tamu.edu).

Color versions of one or more of the figures in this paper are available online at http://ieeexplore.ieee.org.

Digital Object Identifier 10.1109/JSEN.2008.917483

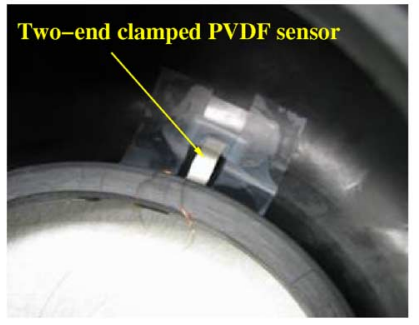

(a)

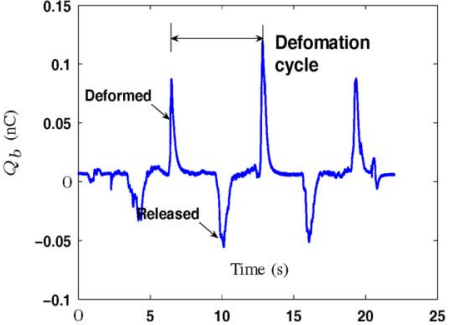

(b)
Fig. 1. PVDF-based tire tread deformation sensor [12]. (a) Two end-clamped sensor along the tire sidewall. (b) Sensor output under a periodic vertical loading.

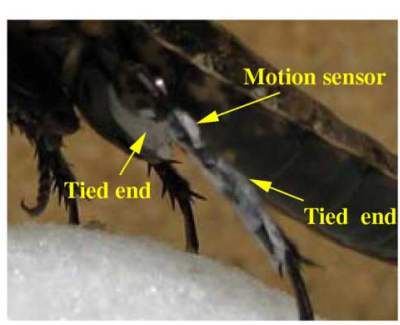

(a)

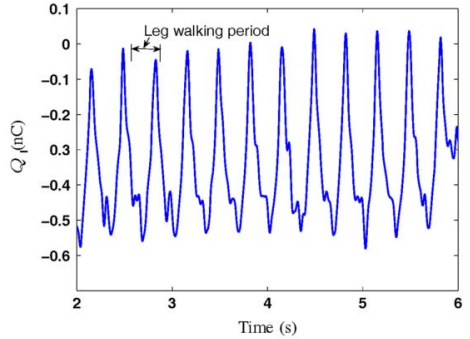

(b)
Fig. 2. PVDF-based piezo sensor for insect locomotion study [13]. (a) The sensor is tied to a pair of legs of an American cockroach. (b) The sensor output when the cockroach is walking.

deformation [10]. In [11] and [12], the use of PVDF-based microsensor to measure the tread deformation has been presented. Fig. 1(a) shows the two-end glued PVDF sensor of a size of $20 \mathrm{~mm} \times 3 \mathrm{~mm} \times 110 \mu \mathrm{m}$ along the sidewall of a robotic tire and Fig. 1(b) shows the sensor output under a stationary cyclic loading. It is clearly seen that the sensor measurements correspond to the radial deformation of the tire. A detailed discussion for the tire sensing system, including the wireless data transmission module, can be found in [6], [11], and [12].

Another application of the PVDF-based deformation piezosensor is for insect locomotion studies. Bioinspired robotic development has been an active research area in both robotics and systems biology communities. Insects, such as American cockroaches, are examples of effective and efficient locomotion mechanisms in nature. For fully understanding the kinematics and biomechanics of insect locomotion, nondistractive in situ motion and force sensor structures must be designed without interfacing with insect running. In [13], a PVDF-based thin-film sensor is reported to measure the leg locomotion of a cockroach. Fig. 2 shows such a development. A custom-built PVDF sensor 
of size $10 \mathrm{~mm} \times 1 \mathrm{~mm} \times 28 \mu \mathrm{m}$ is tied to a pair of legs of a cockroach [Fig. 2(a)] and the sensor output is connected through a thin wire $(0.3 \mathrm{~mm}$ diameter) to an on-board charge amplifier for voltage measurements. Fig. 2(b) shows the sensor output when the cockroach is walking. Some results have shown a correlation between the sensor output and the insect locomotion [13].

In both applications discussed above, the PVDF sensors are clamped at two ends. Under the relative movement of the two sensor ends, the PVDF thin-film is buckled and sensors generate electric charge due to bending motion. Such a new sensing design is different with most PVDF-based sensing applications. Most PVDF-based applications utilize the high piezoelectricity and reliability of the PVDF material for sensor design [14]. In these applications, the PVDF sensor film is glued on or embedded inside the sensed objects and typically the deformation is not large. For large deformation applications, such as vibration sensing in [2], the PVDF film is clamped on a fixed foundation. To the authors' knowledge, besides the two applications that we mentioned in this paper, there is few discussions for PVDF-based large deformation and motion sensing and measurement.

The goal of this paper is to develop and validate an analytical model for the PVDF-based deformation and movement sensors. The analytical models will provide a quantitative relationship between the sensor measurements and the deformation or motion information of the two sensor ends and, thus, facilitate the sensor applications. There are a large amount of research work that study the constitutive relationship between the mechanical forces (moments) and electrical voltage (charge) for multilayer beamed piezoelectric materials. Readers can refer to [14] and [15] and the references therein. We cannot, however, apply the existing analytical results to model the PVDF-based large deformation sensors in our applications for several reasons. First, most modeling work of piezoelectric materials are based on beam theory and small deflections or deformations are typically the underlying assumptions. Second, in most piezo-based sensing applications, the sensors are either glued on surfaces or embedded inside mechanical or civil structures, while for our applications only two ends of the PVDF sensors are attached to moving objects and the main body of the PVDF thin-film are freely deformed.

We take a different approach to consider the PVDF sensor as a thin strip for modeling purposes because of the high aspect ratios in geometry (thin and long) of the sensor design. Typical Euler-type buckling models cannot capture the deformation and shape of such a thin heavy strip. Instead, a heavy elastica model [16], [17] is utilized to describe the deflection of the post-buckling shape of the sensor film. We consider the strain (stress) distribution among the PVDF elastica under the buckling motion and the generated electric charge is then estimated using the constitutive piezoelectric relationship [18]. A hysteresis model is employed to capture the phase-shift nonlinear phenomena in piezoelectric materials. We finally develop a testing kit to validate the sensor modeling and analysis.

The remainder of this paper is organized as follows. In Section II, we present an analytical model for the PVDF sensors under a buckling motion. Experimental comparison and model validation of the deformation sensors are presented

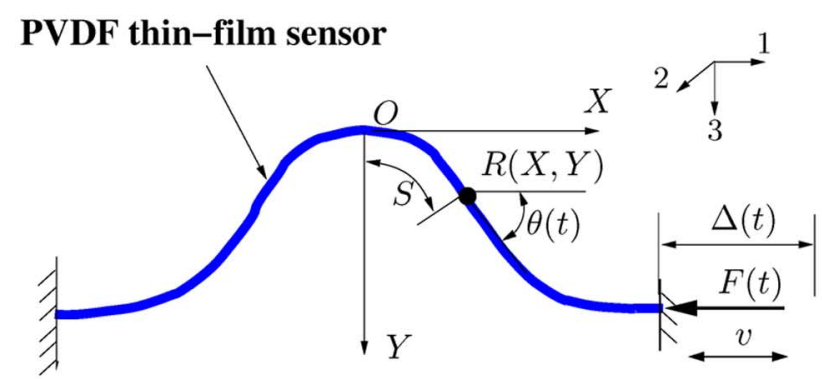

(a)

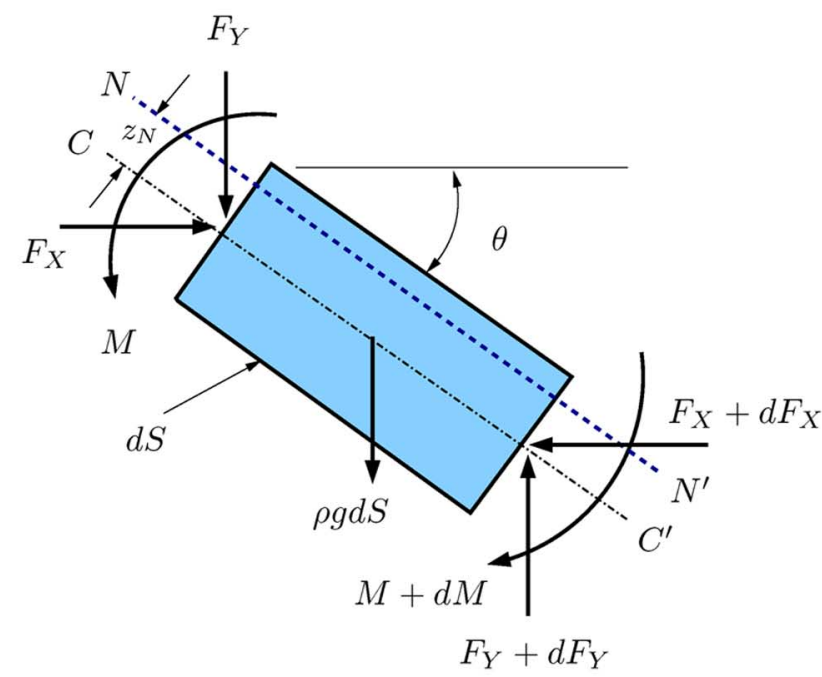

(b)

Fig. 3. (a) A schematic of the buckling shape of a PVDF based heavy elastica sensor. (b) A free body diagram of the differential element of the elastica.

in Section III. The concluding remarks and future research directions are included in Section IV.

\section{SENSOR MODELS}

In this section, we first consider the output model for charge $Q_{e n}$ that is purely generated by the mechanical buckling motion of the PVDF film. Then, we present a hysteresis model to capture the nonlinear property of the PVDF piezo-materials. If we denote the hysteresis operator as $\mathcal{H}(\cdot)$, the sensor output charge $Q_{e}$ is considered as $Q_{e}=\mathcal{H}\left(Q_{e n}\right)$.

\section{A. Sensor Buckling Motion and Charge Calculation}

For the buckling sensors shown in Figs. 1 and 2, we denote the sensor size as $2 L$ (length), $b$ (width), and $t_{S}$ (height), respectively. We consider a PVDF-based buckling elastica shown in Fig. 3(a). One end of the PVDF elastica is clamped at a fixed platform and the other end is clamped with a moving platform. The deformation of the moving end is denoted as $\Delta(t)$ and the applied force is denoted as $F(t)$. We here consider that the deflection $\Delta(t)$ is measured from the position where the PVDF thin-film is flat, namely, initially the PVDF thin-film is flat and $\Delta(0)=0$.

We choose the $X Y$ coordinate with the origin $O$ at the middle point of the PVDF elastica. The deflection at point $R(X, Y)$ is parameterized by the arc-length $S$ (with $S=0$ at point $O)$, as shown in Fig. 3(a). The deflection angle $\theta(S)$ is defined as the tangent directional angle with the $X$ axis. Fig. 3(b) 
shows the free body diagram of a differential element $d S$ at point $R$. The internal forces along the $X$ and $Y$ directions on the differential element $d S$ at time $t$ are denoted as $F_{X}(S, t)$ and $F_{Y}(S, t)$, respectively. The bending moment at point $R$ is denoted as $M(S, t)$ at time $t$. Similar to the developments in [16] and [17], we obtain the motion equations for the PVDF elastica as ${ }^{1}$

$$
\begin{aligned}
\frac{\partial X}{\partial S} & =\cos \theta, \frac{\partial Y}{\partial S}=\sin \theta, \frac{\partial \theta}{\partial S}=\kappa=\frac{M}{E I} \\
\frac{\partial F_{X}}{\partial S} & =-\rho \frac{\partial^{2} X}{\partial t^{2}}, \frac{\partial F_{Y}}{\partial S}=-\rho g-\rho \frac{\partial^{2} Y}{\partial t^{2}}, \\
\frac{\partial M}{\partial S} & =F_{Y} \cos \theta-F_{X} \sin \theta
\end{aligned}
$$

where $E$ is Young's modulus of the PVDF film, $I$ is the second moment inertia of the cross sectional area of the elastica with respect to the bending axis, $\kappa=M / E I$ is the curvature, and $\rho$ is the mass density per unit length of the PVDF strip.

Before we proceed to put (1) into dimensionless equations, we first determine the electric charge $Q_{e n}$ due to the sensor buckling motion. The accumulated electric charge $Q_{e n}$ can be calculated as [18]

$$
Q_{e n}=\int_{A} \mathcal{D}_{3} d A=d_{31} c_{11} \int_{A} \varepsilon_{1} d A
$$

where $\mathcal{D}_{3}$ is electric displacement along the 3 (vertical) direction, $\varepsilon_{1}$ is the strain along the 1 (longitudinal) direction, $A$ is the electrode area of the strip surface, $d_{31}$ is the PVDF piezoelectric constant between the 3 and 1 directions, $c_{11}$ is the stiffness constant of the PVDF film along the 1 direction. For the definition of the strain (stress) and electrical fields direction for the piezo-material [the 123 axes shown in Fig. 3(a)], readers can refer to [18] and [19].

We consider that the electric charge is mainly generated by the bending of the PVDF film. For the differential PVDF element shown in Fig. 3(b), the element is under both bending and axial force and the neutral axis $\left[N N^{\prime}\right.$ in Fig. 3(b)] is no longer the film centriodal axis $\left[C C^{\prime}\right.$ in Fig. 3(b)]. Denote the distance between $N N^{\prime}$ and $C C^{\prime}$ as $z_{N}$ at location $S$ and time $t$. Then, the axial force $F_{a}$ along the sensor film is given by

$$
F_{a}=F_{X} \cos \theta+F_{Y} \sin \theta
$$

and the bending moment is $M$. Note that the strain $\varepsilon=0$ along $N N^{\prime}$ and using the superposition of axial force and bending moment effects, we can calculate $z_{N}$ as

$$
z_{N}=\frac{1}{12} \frac{F_{a}}{M} t_{S}^{2}=\frac{1}{12} \frac{F_{X} \cos \theta+F_{Y} \sin \theta}{M} t_{S}^{2} .
$$

Then, the strain $\varepsilon_{1}$ of the PVDF film is calculated as

$$
\begin{aligned}
\varepsilon_{1} & =z_{N} \kappa=z_{N} \frac{M}{E I} \\
& =\frac{F_{X} \cos \theta+F_{Y} \sin \theta}{E b t_{S}}
\end{aligned}
$$

${ }^{1}$ For notation simplicity, we drop the variable dependence on arc-length $S$ and time $t$ in the rest of this paper. where we use $I=(1 / 12) b t_{S}^{3}$ for a rectangular cross section area. Due to the symmetry of the film, we only consider the half portion $S>0$, and thus write (2) as

$$
Q_{e n}=\frac{2 d_{31} c_{11}}{E t_{S}} \int_{0}^{L}\left(F_{X} \cos \theta+F_{Y} \sin \theta\right) d S .
$$

We define the following nondimensional quantities:

$$
\begin{aligned}
& x=\frac{X}{L}, y=\frac{Y}{L}, s=\frac{S}{L}, \delta=\frac{\Delta}{L}, \\
& m=\frac{M L}{E I}, f=\frac{F_{X} L^{2}}{E I}, q=\frac{F_{Y} L^{2}}{E I}, \\
& w=\frac{\rho g L^{3}}{E I}, \tau=\frac{t}{L^{2}} \sqrt{\frac{E I}{\rho}}, \Omega=\omega L^{2} \sqrt{\frac{\rho}{E I}}
\end{aligned}
$$

where $\omega$ is the motion frequency. With the nondimensional quantities, (1) can be written as

$$
\begin{aligned}
\frac{\partial x}{\partial s} & =\cos \theta, \frac{\partial y}{\partial s}=\sin \theta, \frac{\partial \theta}{\partial s}=m, \\
\frac{\partial f}{\partial s} & =-\frac{\partial^{2} x}{\partial \tau^{2}}, \frac{\partial q}{\partial s}=-w-\frac{\partial^{2} y}{\partial \tau^{2}}, \\
\frac{\partial m}{\partial s} & =q \cos \theta-f \sin \theta .
\end{aligned}
$$

Furthermore, we separate the variables as the sum of equilibrium and dynamic components, namely

$$
\begin{aligned}
x(s, \tau) & =x_{e}(s)+x_{d}(s) \sin (\Omega \tau) \\
y(s, \tau) & =y_{e}(s)+y_{d}(s) \sin (\Omega \tau) \\
\theta(s, \tau) & =\theta_{e}(s)+\theta_{d}(s) \sin (\Omega \tau) \\
m(s, \tau) & =m_{e}(s)+m_{d}(s) \sin (\Omega \tau) \\
f(s, \tau) & =f_{e}(s)+f_{d}(s) \sin (\Omega \tau) \\
q(s, \tau) & =q_{e}(s)+q_{d}(s) \sin (\Omega \tau)
\end{aligned}
$$

where the symbols with subscriptions " $e$ " represent the (time independent) equilibrium components and symbols with subscriptions " $d$ " represent the dynamic components that are part of time-dependent terms.

We then write the equations that the equilibrium components must be satisfied as

$$
\begin{aligned}
\frac{d x_{e}}{d s} & =\cos \theta_{e}, \frac{d y_{e}}{d s}=\sin \theta_{e}, \frac{d \theta_{e}}{d s}=m_{e} \\
\frac{d m_{e}}{d s} & =q_{e} \cos \theta_{e}-f_{e} \sin \theta_{e} .
\end{aligned}
$$

The normalized force $q_{e}$ satisfies $q_{e}(s)=s w$ since $q_{e}$ is the normalized gravity force from $S=0$. The boundary conditions for (7) are

$$
\begin{aligned}
& x_{e}(0)=y_{e}(0)=\theta_{e}(0)=0 \\
& x_{e}(1)=1-\delta, \theta_{e}(1)=0 .
\end{aligned}
$$

We consider the charge generated by the quasi-static equilibria and, therefore, we write (5) as

$$
Q_{e n}=2 d_{31} c_{11} \frac{I}{L t_{S}} \int_{0}^{1}\left(f_{e} \cos \theta_{e}+q_{e} \sin \theta_{e}\right) d s .
$$




\section{B. Charge Approximation}

The exact analytical solutions for the differential (7) with the boundary conditions (8) cannot be obtained [16]. Therefore, we instead approximate the solutions for the boundary-value problem given by (7) and (8).

For a small deflection $\theta \ll 1$ and the fact that the flexural rigidity $E I$ is a dominant factor for the PVDF strip, we assume $w=\epsilon^{3} \ll 1$ for a small $\epsilon>0$. We then approximate each equilibrium component in (7) as a perturbation of $\epsilon$ terms

$$
\begin{aligned}
\theta_{e}(s) & =\epsilon \theta_{0}(s)+\epsilon^{3} \theta_{1}(s)+O\left(\epsilon^{5}\right), \\
x_{e}(s) & =x_{0}(s)+\epsilon^{2} x_{1}(s)+O\left(\epsilon^{4}\right), \\
y_{e}(s) & =\epsilon y_{0}(s)+\epsilon^{3} y_{1}(s)+O\left(\epsilon^{5}\right), \\
f_{e}(s) & =f_{0}(s)+\epsilon^{2} f_{1}(s)+O\left(\epsilon^{4}\right), \\
\sin \theta_{e} & =\epsilon \theta_{0}+\frac{1}{6} \epsilon^{3}\left(-\theta_{0}^{3}+6 \theta_{1}\right)+O\left(\epsilon^{5}\right), \\
\cos \theta_{e} & =1+\frac{1}{2} \epsilon^{2}\left(-\theta_{0}^{2}\right)+O\left(\epsilon^{4}\right) .
\end{aligned}
$$

Here, we consider the fact that $\theta_{e}, y_{e}$, and $\sin \theta_{e}$ are small and, thus, as a sum of odd-power terms of $\epsilon$ and $x_{e}$, and $\cos \theta_{e}$ are as a sum of even-power terms of $\epsilon$ [16].

Substituting the above approximations into (7) and taking the first two terms in $\epsilon$ in each equation, we obtain

$$
\begin{aligned}
\frac{d x_{0}}{d s} & =1, \frac{d x_{1}}{d s}=-\frac{1}{2} \theta_{0}^{2}, \frac{d y_{0}}{d s}=\theta_{0}, \\
\frac{d y_{1}}{d s} & =\theta_{1}-\frac{1}{6} \theta_{0}^{3}, \frac{d^{2} \theta_{0}}{d s^{2}}=-f_{0} \theta_{0}, \\
\frac{d^{2} \theta_{1}}{d s^{2}} & =s-f_{1} \theta_{0}-f_{0} \theta_{1}+\frac{1}{6} f_{0} \theta_{0}^{3} .
\end{aligned}
$$

The boundary conditions given by (8) can be rewritten as

$$
\begin{gathered}
x_{0}(0)=x_{1}(0)=0, y_{0}(0)=y_{1}(0)=0, \\
\theta_{0}(0)=\theta_{1}(0)=0, \theta_{0}(1)=\theta_{1}(1)=0 .
\end{gathered}
$$

We can solve (10) with the above boundary conditions and obtain solutions for $\theta_{0}$ and $\theta_{1}$ as

$$
\begin{aligned}
& \theta_{0}=C \sin (\pi s) \\
& \theta_{1}=\frac{C^{3}}{192} \sin (3 \pi s)+\frac{s}{\pi^{2}}[1+\cos (\pi s)]
\end{aligned}
$$

where constant $C$ is determined by the external force $F$. With $\theta_{0}$ and $\theta_{1}$, we can obtain the normalized force $f_{e}$ from (10) as

$$
f_{0}=\pi^{2}, f_{1}=\frac{\pi^{2} C^{2}}{8}+\frac{2}{\pi C}
$$

and

$$
f_{e}=\pi^{2}+\epsilon^{2}\left(\frac{\pi^{2} C^{2}}{8}+\frac{2}{\pi C}\right)+O\left(\epsilon^{4}\right) .
$$

For $x_{0}, x_{1}$, and $y_{0}$, we have

$$
\begin{aligned}
& x_{0}=s, x_{1}=\frac{C^{2}}{4}\left[\frac{1}{2 \pi} \sin (2 \pi s)-s\right] \\
& y_{0}=\frac{C}{\pi}[1-\cos (\pi s)] .
\end{aligned}
$$

The normalized deflection $\delta$ of the moving end of the PVDF elastica and the maximum height of the PVDF elastica deflection $h_{\max }$ can be calculated as

$$
\delta=\frac{1}{4} C^{2} \epsilon^{2}+O\left(\epsilon^{4}\right)
$$

and

$$
h_{\max }=\frac{2}{\pi} C \epsilon+O\left(\epsilon^{3}\right) .
$$

Using (12) and (13), we approximately calculate the electric charge (9) as

$$
\begin{aligned}
Q_{\text {en }}=2 d_{31} c_{11} \frac{I}{L t_{S}} \int_{0}^{1}\{ & {\left[\pi^{2}+\epsilon^{2}\left(\frac{\pi^{2} C^{2}}{8}+\frac{2}{\pi C}\right)\right] } \\
& {\left.\left[1+\frac{1}{2} \epsilon^{2}\left(-C^{2} \sin ^{2} \pi s\right)\right]\right\} d s }
\end{aligned}
$$

where in the above approximation, we drop the high-order terms that contain $O\left(\epsilon^{4}\right)$. Using the relationship $\delta=(1 / 4) C^{2} \epsilon^{2}$, we simplify (14) as

$$
\begin{aligned}
Q_{e n} & \approx 2 d_{31} c_{11} \frac{I}{L t_{S}} \pi^{2}\left(1+\frac{1}{2} \delta\right)(1-\delta) \\
& =K\left(1+\frac{1}{2} \delta\right)(1-\delta)
\end{aligned}
$$

where $K=2 d_{31} c_{11}\left(I / L t_{S}\right) \pi^{2}$ is a constant. It is noted that the output charge by the piezoelectric material, such as PVDF, only responses to the dynamic deflection motion $\delta$. Therefore, the actual output charge should filter out the dc component in the above approximation given in (15), namely

$$
Q_{e n}=-\frac{K}{2} \delta-\frac{K}{2} \delta^{2}
$$

and if the deflection is small, i.e., $\delta \ll 1$, we can further approximate the charge $Q_{e n}$ as

$$
Q_{e n} \approx-\frac{K}{2} \delta
$$

Equation (16) implies that the charge generated by the dynamic deflection $\delta$ is proportional to the deflection magnitude. However, there exists a phase shift between the output charge $Q_{e}(t)$ and the deflection $\Delta(t)=L \delta(t)$. We are now ready to discuss how to capture such a nonlinear phase-shift effect.

\section{Hysteresis Modeling}

The existence of hysteresis between sensor input and output is widely known for piezoelectric sensors [19]. Fig. 4 show the experimental results for a buckling test of a PVDF sensor of a size of $32 \mathrm{~mm} \times 3 \mathrm{~mm} \times 110 \mu \mathrm{m}$ under a sinusoidal deflection $\Delta(t)=4[-1+\sin (10 \pi t)] \mathrm{mm}$. Fig. 4(a) shows the time-trajectory and Fig. 4(b) shows a hysteresis curve between charge $Q_{e}(t)$ and deflection $\Delta(t)$ due to their phase differences.

We consider a Duhem model to capture the rate-independent hysteresis relationship between the charge outputs $Q_{e}$ and $Q_{e n}$ [20], [21]. Here, we consider the buckling-generated charge $Q_{e n}$ as the input to the hysteresis operator, namely, $Q_{e}=\mathcal{H}\left(Q_{e n}\right)$. 


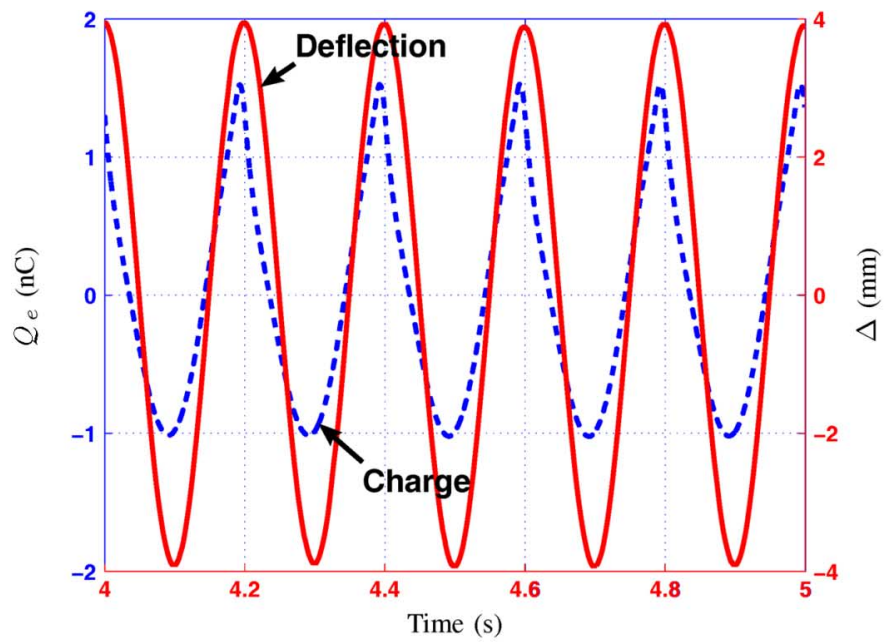

(a)

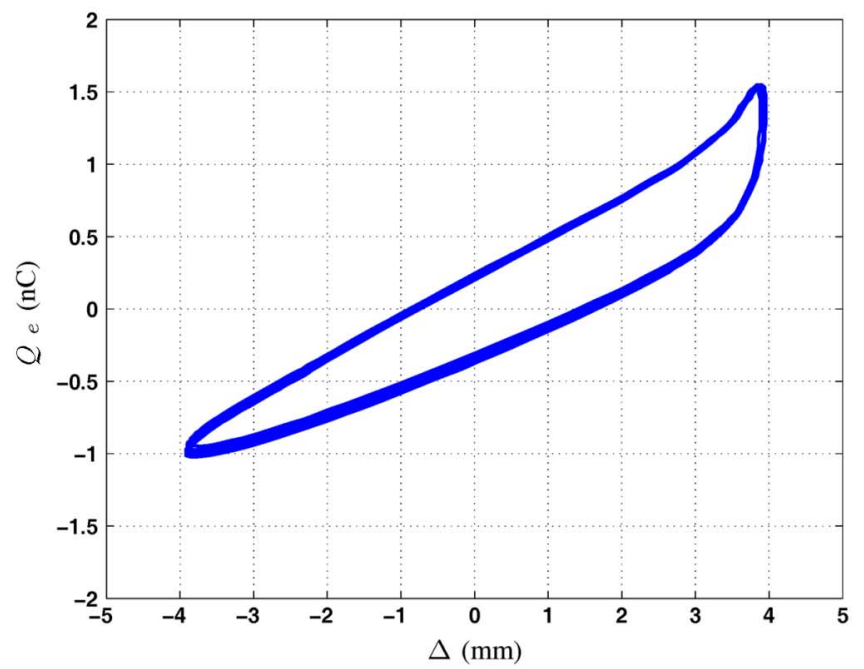

(b)

Fig. 4. (a) Measured charge output and the cyclic deflection $\Delta(t)=4[-1+$ $\sin (10 \pi t)] \mathrm{mm}$. (b) A hysteresis curve between the charge $Q_{e}$ and deflection $\Delta$.

The Duhem model represents operator $\mathcal{H}(\cdot)$ by a first-order nonlinear differential equation as

$$
\dot{Q}_{e}=\alpha\left|\dot{Q}_{e n}\right|\left(a Q_{e n}-Q_{e}\right)+b \dot{Q}_{e n}
$$

where $\alpha>0$ and $a>b \geq(1 / 2) a$ are model constants that depend on the shape and area of the experimental hysteresis curves. In (17), the output charge $Q_{e}$ is considered as the state variable of the differential equation and is dependent on the values of both $Q_{e n}$ and $\dot{Q}_{e n}$. Such a mathematical relationship (17) can reproduce the hysteresis phenomena that we observe in experiments. Readers can refer to [21] for more details on how to estimate these hysteresis model parameters.

It is noted that due to the existing hysteresis in PVDF piezoelectric materials, the output charge $Q_{e}$ is a nonlinear function of the deflection $\Delta(t)$ although the calculated charge $Q_{e n}$ due to the buckling motion is linear in $\Delta(t)$, as shown in (16). In the next section, we will discuss experiments and estimation of model parameters for sensor output charge models.

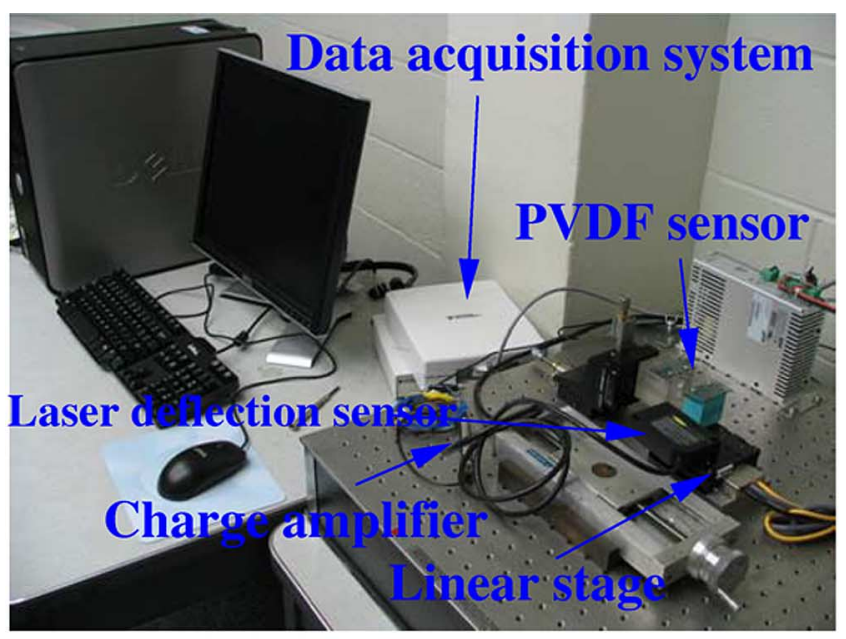

(a)

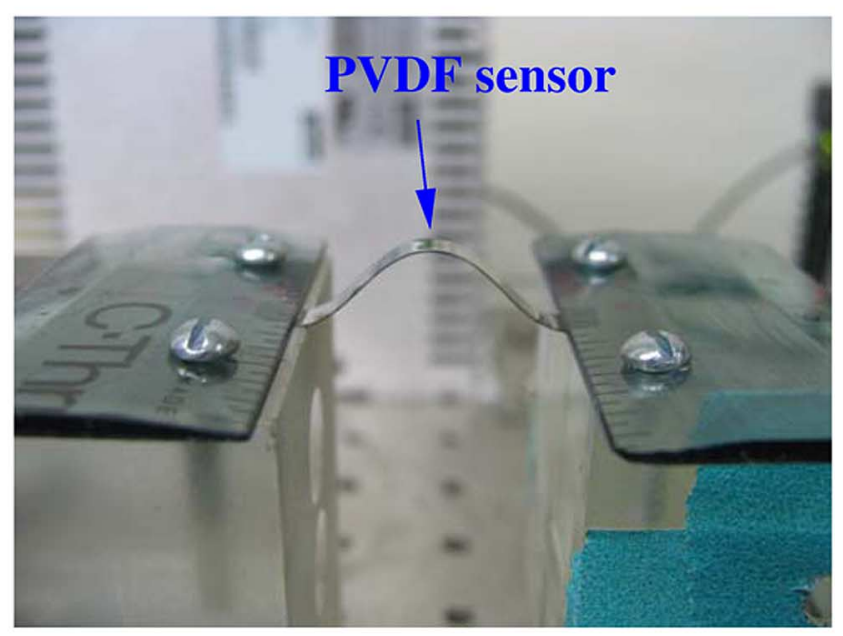

(b)

Fig. 5. (a) A experiment setup for buckling sensor testing. (b) A buckled PVDF sensor.

\section{EXPERIMENTS}

\section{A. Experiment Setup}

We have developed a deformation sensor characteristic testing kit to experimentally capture and validate the relationship between the sensor output charge and the deflection. Fig. 5 shows the experiment setup. We use several types of metalized PVDF thin films (from Measurement Specialties, Inc.) with different thicknesses. The PVDF thin-film is fabricated into different sizes. Thin wires are connected to one end of the sensor by conductive glue. A computer-controlled linear stage (Parker MX80S) is used to move one end of the PVDF sensor film to simulate the deflection motion, while the other end of the sensor is clamped at a stationary fixture. A laser deflection sensor (Banner LG5) is employed to monitor the deflection of the PVDF thin-film sensor at a fixed location. The linear stage controller is implemented using a high-performance servo control board (NI PCI 7350) from National Instruments, Inc. The controlled motion trajectory is designed through NI LabView. A data acquisition system (NI PCI 6221) records 


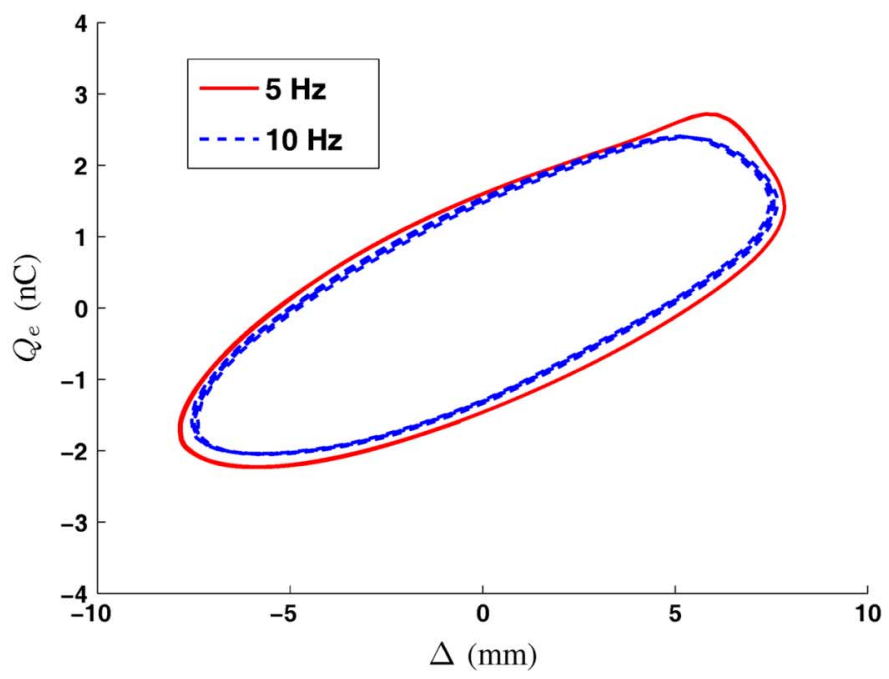

(a)

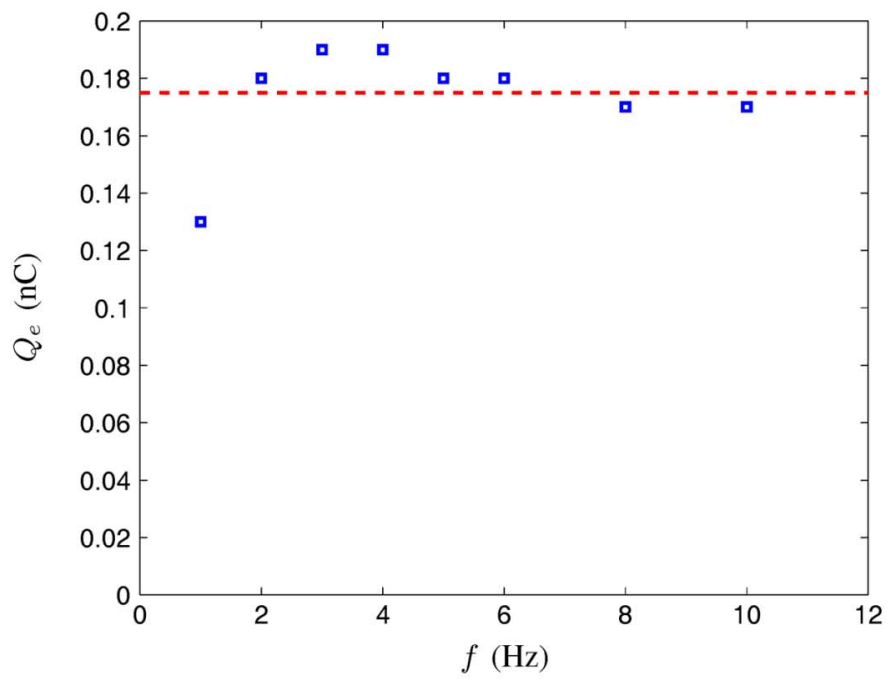

(b)

Fig. 6. (a) The output charge of a PVDF sensor under a sinusoidal deflection motion with frequencies of 5 and $10 \mathrm{~Hz}$ and a peak amplitude of $8 \mathrm{~mm}$ for a PVDF sensor of a size of $32 \mathrm{~mm} \times 3 \mathrm{~mm} \times 110 \mu \mathrm{m}$. (b) Output charge peak values $Q_{e}$ versus excitation frequency $f$ under a sinusoidal deflection motion with a peak amplitude of $2 \mathrm{~mm}$ for a PVDF sensor of a size of $10 \mathrm{~mm} \times 1$ $\mathrm{mm} \times 28 \mu \mathrm{m}$.

sensor output and feedback motion information. The sensor output is conditioned by a charge amplifier (PCB 422E03) before the data is sampled through the data acquisition system.

\section{B. Experiment Results}

In all experiments, we choose that the peak-to-peak amplitude of the sinusoidal deflection $\Delta(t)$ is around $50 \%$ of the sensor length $2 L$. We first test the PVDF sensor under buckling with various motion frequencies. Fig. 6(a) shows the output charge $Q_{e}$ as a function of the deflection $\Delta(t)$ under a sinusoidal motion of two frequencies. The PVDF sensor is of a size of 32 $\mathrm{mm} \times 3 \mathrm{~mm} \times 110 \mu \mathrm{m}$ and the peak amplitude of the deflection is $8 \mathrm{~mm}$ (peak-to-peak amplitude is $16 \mathrm{~mm}$ ). From Fig. 6(a), it is clearly observed a fixed hysteresis pattern under both frequencies. We also take a smaller size PVDF sensor $(10 \mathrm{~mm} \times 1$ $\mathrm{mm} \times 28 \mu \mathrm{m})$ and measure the sensor output under a sinusoidal

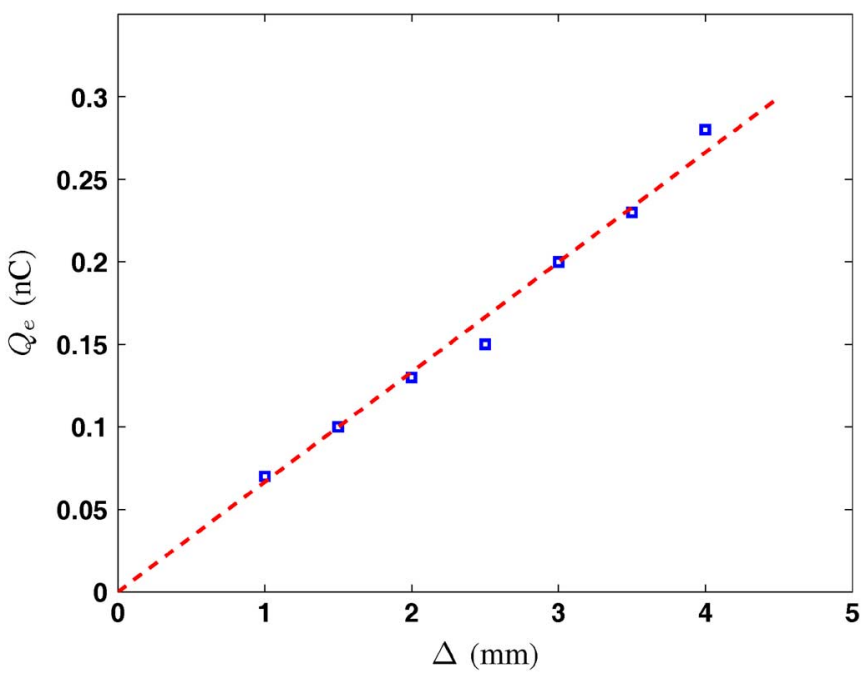

Fig. 7. Output charge peak values $Q_{e}$ versus deflection peak amplitudes under a $2 \mathrm{~Hz}$ sinusoidal motion for a PVDF sensor of a size of $10 \mathrm{~mm} \times 1 \mathrm{~mm} \times 28 \mu \mathrm{m}$.

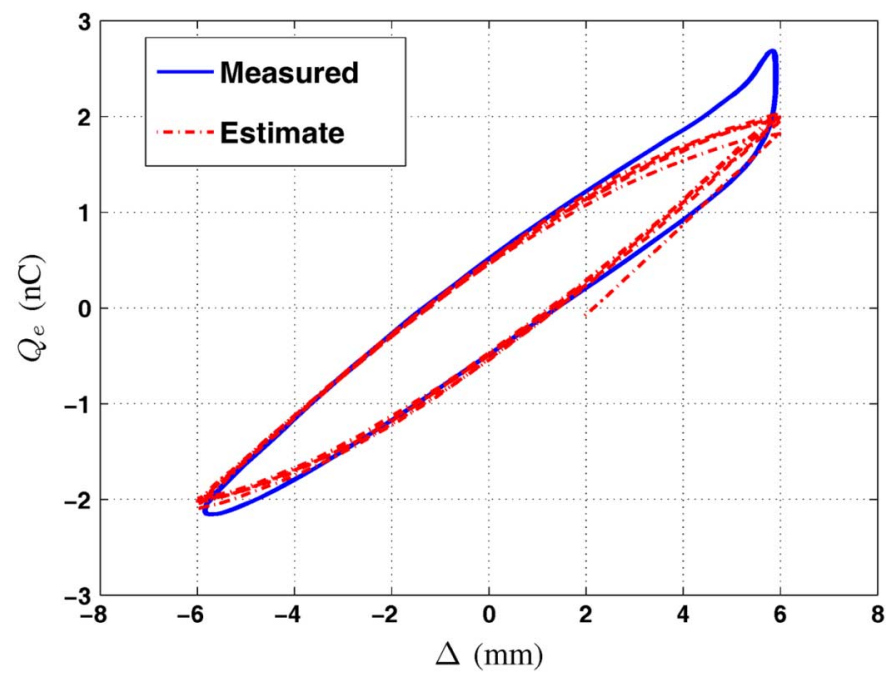

Fig. 8. Measured and estimated hysteresis curves for a PVDF sensor of a size of $25 \mathrm{~mm} \times 3 \mathrm{~mm} \times 110 \mu \mathrm{m}$ under a $5 \mathrm{~Hz}$ and $6 \mathrm{~mm}$ peak amplitude sinusoidal deflection motion.

deflection of a peak amplitude of $2 \mathrm{~mm}$ (peak-to-peak amplitude is $4 \mathrm{~mm}$ ) with various frequencies. Fig. 6(b) illustrates that the peak charge values are kept constantly under a varying excitation frequency from 1 to $10 \mathrm{~Hz}$. These results confirm that the hysteresis generated by PVDF thin-film is independent of the time scale of the input excitation and thus rate-independent.

Fig. 7 shows that the peak values of the sensor output charge are proportional to the deflection peak amplitude. Such an observation validates our analysis of the output charge as given by (16). Note that the linear correlation between the magnitudes of output charge $Q_{e}(t)$ and deflection $\Delta(t)$ only shows their peak value relationship. Due to the existing hysteresis, the instantaneous relationship between $Q_{e}(t)$ and $\Delta(t)$ is highly nonlinear and in the following we are going to estimate the hysteresis model.

Fig. 8 shows a comparison between the measured and Duhem model-based estimated hysteresis curves between $Q_{e}(t)$ and 
TABLE I

Estimated PARAMETERS OF THE DUHEM HySTERESIS MODEL AND SENSOR MODEL

\begin{tabular}{|l|l|l|l|}
\hline$\alpha\left(\mathrm{nC}^{-1}\right)$ & $a$ & $b$ & $K(\mathrm{nC})$ \\
\hline 0.15 & 0.54 & 0.28 & 34.47 \\
\hline
\end{tabular}

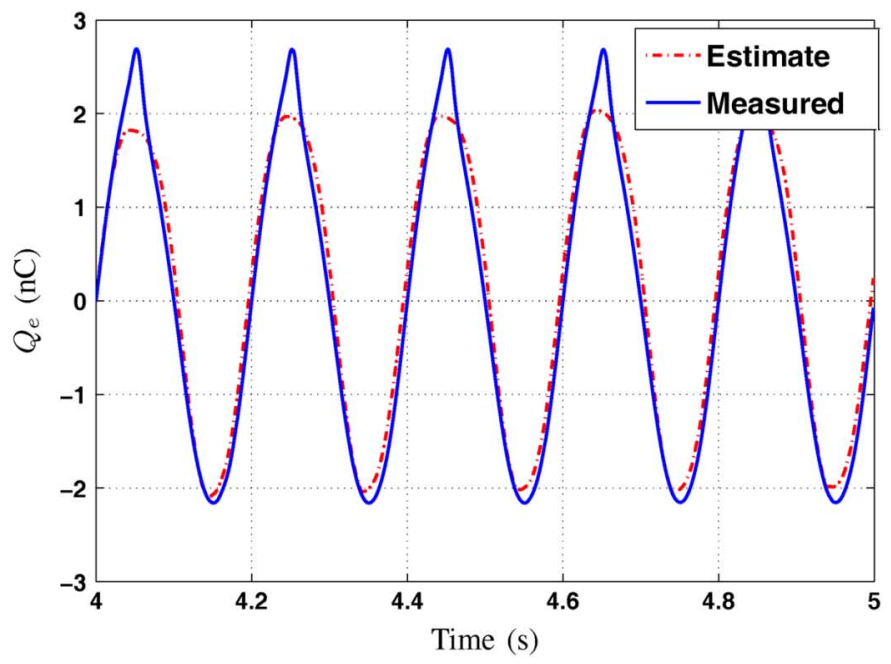

(a)

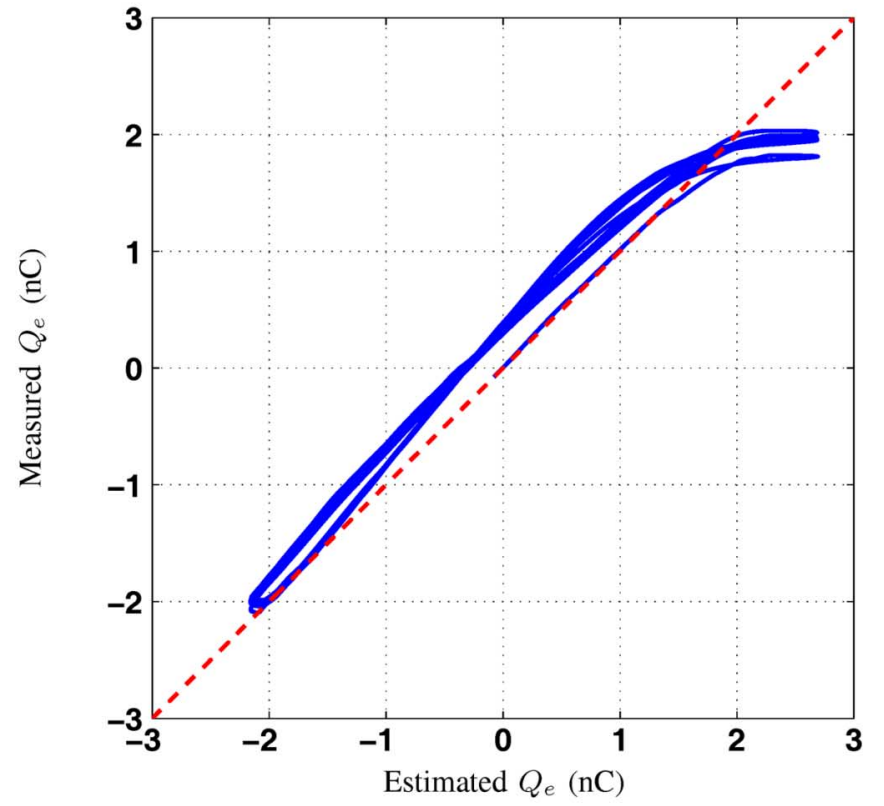

(b)

Fig. 9. (a) Measured and model estimated charge values $Q_{e}(t)$ for a PVDF sensor of a size of $25 \mathrm{~mm} \times 3 \mathrm{~mm} \times 110 \mu \mathrm{m}$ under a sinusoidal deflection motion at a frequency of $5 \mathrm{~Hz}$ and with a peak amplitude of $6 \mathrm{~mm}$. (b) Model-based estimated charge versus measurement.

$\Delta(t)$. The PVDF sensor in the estimation is of a size of $25 \mathrm{~mm} \times 3 \mathrm{~mm} \times 110 \mu \mathrm{m}$ and the deflection is in a sinusoidal form with a frequency of $5 \mathrm{~Hz}$ and a peak amplitude of $6 \mathrm{~mm}$ (a peak-to-peak amplitude is $12 \mathrm{~mm}$ ). The Duhem hysteresis model parameters are listed in Table I. Those parameters are obtained from the experiments by the relationships given in [21]. The estimated hysteresis curve fits well with the measurements. Note that at the positive peak values in the experimental measurement, the sensor has been pulled slightly by the linear stage and, thus, we observe a "sharp" peak in each cycle. It is also noted that in Fig. 8 the first few cycles in the estimated curves are in transient and not consistent with steady-state curves due to the use of a zero initial condition in the Duhem model.

Fig. 9 shows the comparison of model-based estimates of the output charge with the measurements. It is again shown in Fig. 9(a) that there is a sharp peak at the positive cycle due to the pull effect of the linear stage in experiments. A comparison plot of the estimated versus measured charge values shown in Fig. 9(b) demonstrates a linear relationship with a slope of value of one. These results validate that the hysteresis model-based estimation captures the sensor measurements within a small error range.

\section{CONCLUSION}

The use of the PVDF-based buckling sensor for deformation and motion measurements discussed in this paper is motivated by the applications of tire rubber deformation and insect locomotion studies. By sensing mechanism design in these applications, the PVDF sensors are under a cyclic buckling motion, and only two ends are attached to sensed object. In this paper, we presented the mathematical modeling, analysis, and experimental validation for such PVDF sensors. The modeling approach was taken into two steps: we first treated PVDF thin-film as a heavy elastica strip and a sensor model was developed. We found that for a small deflection, the magnitude of the sensor output charge is proportional to the deflection magnitude. However, there is a phase shift between the charge and motion deflection and a hysteresis pattern has been observed. A Duhem hysteresis model was then used to capture such a nonlinear relationship between the charge and the deflection. The integrated model of the elastica buckling and hysteresis analysis was estimated and validated by experiments on a computer-controlled linear motion-stage testbed.

We plan to extend the current research into several directions. We like to integrate a dynamic hysteresis inverter to compensate the nonlinear hysteresis effect and, thus, build a linear relationship between the arbitrary input deflection and sensor measurements. We also plan to refine our modeling development and apply the refined sensing models for the two applications mentioned in this paper. We will report these developments in the future.

\section{ACKNOWLEDGMENT}

The authors thank B. Mika for setting up the experimental apparatus and collecting all test data. They are also grateful to Prof. D. Song, Prof. R. Langari, and Prof. S. Khatri at Texas A\&M University for helpful discussions.

\section{REFERENCES}

[1] B. Li and V. Giurgiutiu, "Modeling and testing of pzt and PVDF piezoelectric wafer active sensors," Smart Mat. Struct., vol. 15, pp. 1085-1093, 2006.

[2] M. Toda and M. Thompson, "Contact-type vibration sensors using curved clamped PVDF film," IEEE Sensors J., vol. 6, no. 5, pp. 1170-1177, 2006.

[3] R. Marsili, "Measurement of the dynamic normal pressure between tire and ground using PVDF piezoelectric films," IEEE Trans. Instrum. Meas., vol. 49, no. 4, pp. 736-740, Aug. 2000. 
[4] S. K. Kim, I. Cho, J. H. Lee, J. Park, D. H. Yi, and D. Cho, “A new method for accurately estimating the weight of moving vehicles using piezoelectric sensors and adaptive-footprint tire model," Veh. Syst. Dyn., vol. 39, no. 2, pp. 135-148, 2003.

[5] "Federal motor vehicle safety standard: Tire pressure monitoring systems; controls and displays," National Highway Traffic Safety Administration, Dept. Transportation, 2000, docket No. NHTSA 2000-8572.

[6] J. Yi, "A piezo-sensor based 'smart tire' system for mobile robots and vehicles," IEEE/ASM Trans. Mechatron., vol. 13, no. 1, pp. 95-103, 2008.

[7] APOLLO Consortium, "Intelligent tyre systems - state of the art and potential technologies," Technical Research Centre of Finland (VTT), APOLLO Deliverable D7 for Project IST-2001-34372, 2003.

[8] O. Yilmazoglu, M. Brandt, J. Sigmund, E. Genc, and H. Hartnagel, "Integrated inas/gasb 3D magnetic field sensors for 'the intelligent tire'," Sens. Actuators, A, vol. 94, pp. 59-63, 2001.

[9] A. Pohl, R. Steindl, and L. Reindl, "The 'intelligent tire' utilizing passive saw sensors-measurement of tire friction," IEEE Trans. Instrum. Meas., vol. 48, no. 6, pp. 1041-1046, Aug. 1999.

[10] R. Matsuzaki and A. Todoroki, "Wireless flexible capacitive sensor based on ultra-flexible epoxy resin for strain measurement of automobile tires," Sens. Actuators, A, vol. 140, pp. 32-42, 2007.

[11] K. Moon, H. Liang, J. Yi, and B. Mika, "Tire tread deformation sensor and energy harvester development for 'Smart Tire' applications," in Proc. SPIE, San Diego, CA, 2007, vol. 6529.

[12] J. Yi and H. H. Liang, "Development of a PVDF-based tire tread deformation sensing system," in Proc. 2007 World Forum on Smart Mat. Smart Struct. Tech., Nanjing, China, 2007.

[13] H. Lee, R. Cooper, B. Mika, D. Clayton, R. Garg, J. Gonzales, S. Vinson, S. Khatri, and H. Liang, "Polymeric sensors to monitor cockroach locomotion," IEEE Sensors J., vol. 7, no. 12, pp. 1698-1702, Dec. 2007.

[14] I. Chopra, "Review of state of art of smart structures and integrated systems," AIAA J., vol. 40, no. 11, pp. 2145-2187, 2002.

[15] R. G. Ballas, H. Schlaak, and A. Schmid, "The constituent equations of piezoelectric multilayer bending actuators in closed analytical form and experimental results," Sens. Actuators, A, vol. 130-131, pp. 91-98, 2006.

[16] C. Wang, "On symmetric buckling of a finite flat-lying heavy sheet," ASME J. Appl. Mech., vol. 51, no. 2, pp. 278-282, 1984.

[17] S. Santillan, L. Virgin, and R. Plaut, "Post-buckling and vibration of heavy beam on horizontal or inclined rigid foundation," ASME J. Appl. Mech., vol. 73, no. 4, pp. 664-671, 2006

[18] IEEE Standard on Piezoelectricity, ANSI/IEEE Std. 176-1987, 1987.

[19] V. Giurgiutiu and S. Lyshevski, Micromechatronics: Modeling, Analysis, and Design with MATLAB.. Boca Raton, FL: CRC Press, 2004.
[20] B. Coleman and M. Hodgdon, "A constitutive relation for rate-independent hysteresis in ferromagnetically soft materials," Int. J. Eng. Sci. vol. 24, no. 6, pp. 897-919, 1986.

[21] H. Adriaens, W. de Koning, and R. Banning, "Modeling piezoelectric actuators," IEEE/ASME Trans. Mechatron., vol. 5, no. 4, pp. 331-341, Dec. 2000.

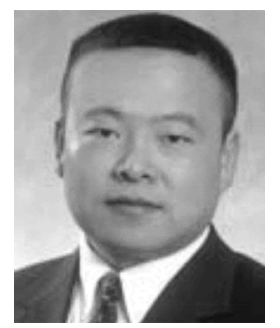

Jingang Yi (S'99-M'02-SM'07) received the B.S. degree in electrical engineering from the Zhejiang University, Hangzhou, China, in 1993, the M.Eng. degree in precision instruments from Tsinghua University, Beijing, China, in 1996, the M.A. degree in mathematics, and the Ph.D. degree in mechanical engineering from the University of California, Berkeley, in 2001 and 2002, respectively.

$\mathrm{He}$ is currently an Assistant Professor of Mechanical Engineering at San Diego State University, San Diego, CA. From May 2002 to January 2005, he was with Lam Research Corporation, Fremont, CA, as a member of Technical Staff. From January 2005 to December 2006, he was with the Department of Mechanical Engineering, Texas A\&M University, as a Visiting Assistant Professor. His research interests include autonomous and robotic systems, dynamic systems and control, intelligent sensing and actuation systems, mechatronics, automation science and engineering, with applications to semiconductor manufacturing and intelligent transportation systems.

Dr. Yi is a member of the American Society of Mechanical Engineers (ASME). He was the recipient of the Kayamori Best Paper Award of the 2005 IEEE International Conference on Robotics and Automation (ICRA) and the Best Conference Paper Finalist of the 2007 IEEE International Conference on Automation Science and Engineering (CASE).

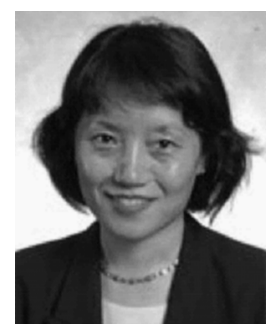

Hong Liang received the Ph.D. degree in materials science from Stevens Institute of Technology, Hoboken, NJ.

She is an Associate Professor and Jordan Career Development Professor with the Department of Mechanical Engineering, Texas A\&M University, College Station. She has more than 120 technical publications including three books. Her research area includes sensor design and materials development, as well as characterization.

Prof. Liang is the recipient of the NSF CAREER Award. She is a Fellow of the Society of Tribologists and Lubrication Engineers. 